\title{
Evolution in Pharmacy Education: Developing Effective Patient Care Practitioners
}

\author{
Lalitha Raman-Wilms
}

$\mathrm{C}$ hanges outlined in the Blueprint for Pharmacy emphasize the need to provide "[o]ptimal drug therapy outcomes for Canadians through patient-centred care". ${ }^{1}$ This approach requires pharmacists to contribute to outcomes-focused patient care, while working within health care teams; pharmacists also need to be accountable and responsible for the safe and effective use of medications.

There is an urgent need for all pharmacists to take on this responsibility. Almost half of patients visiting a community pharmacy or a clinic will have a drug therapy problem. ${ }^{2}$ Patients who have been admitted to hospital also experience a substantial number of preventable adverse drug events. ${ }^{3}$ Medication costs represent the fastest-rising expenditure within our health care system. ${ }^{4}$ Furthermore, with increased use of medications, there is potential for even more adverse drug events to occur. Also, the elderly population uses the most medications, exposing them to an even greater risk of drug therapy problems; in fact, by 2036, 1 in 4 Canadians will be over 65 years of age..$^{5}$ Pharmacists are required to effectively manage drug therapy for all patients, and both faculties of pharmacy and the profession as a whole have obligations to prepare graduating pharmacists for this role.

Education to prepare pharmacists for expanded patient care roles has started to change. For example, all pharmacy schools in Canada have made a commitment to have an entry-level PharmD curriculum in place by $2020 .^{6}$ Some key components of new curricula include an expanded and integrated approach to teaching pharmacotherapy, management courses that include development of patient care services, incorporation of physical assessment and competencies related to the expanded scope of practice (e.g., training for administration of injections), and extensive clinical training in direct patient care. This expanded education is supported nationally by the 2010 educational outcomes of the Association of Faculties of Pharmacy of Canada, which call for pharmacy graduates to be "medication therapy experts". Also, the Canadian Council for Accreditation of Pharmacy Programs (CCAPP) has now approved new standards for PharmD first professional degree programs, which will be implemented in early 2013. Each program must offer a total of
40 weeks (1600 h) of experiential education, with early and mid-program practice experiences lasting at least 8 weeks $(320 \mathrm{~h})$ and concluding practice experiences (at the end of the program) lasting at least 24 weeks $(960 \mathrm{~h})$. This level of clinical training is considered essential for graduates to become independent, competent patient care practitioners, who are responsible to both patients and colleagues within interprofessional teams.

Current training, although effective, is demanding for the preceptor and the site and does not explicitly hold the student accountable to the patient and the rest of the health care team. There are many challenges and just as many opportunities as we consider a paradigm shift in how we train pharmacy students.

At the June 2011 meeting of the American Society of Health-System Pharmacists, Ashby ${ }^{8}$ proposed a pharmacy student training model that would enable trainees to become more accountable and make them indispensable within the patient care team. He also emphasized the importance of students undertaking patient care activities that are known to improve patient outcomes. Additional recommendations included extension of rotational experiences and provision of support to students in their own desired career paths by matching training to interests.

In this issue of the $C J H P$, Hall and others ${ }^{9}$ provide an insightful overview of current experiential training in hospital pharmacy and put forward 8 guiding principles that they consider as key factors for the success of future experiential models. Much of what they outline is akin to training within medical programs, including starting experiential education earlier in the program; providing a mix of hospital and community site experience early, with sustained practices in later years 
(e.g., the Longitudinal Advanced Pharmacy Practice [LAPP] model); incorporating a matching program for LAPP-type rotations; incorporating peer and near-peer learning models of training; including interdisciplinary training; and involving students in activities that result in positive patient outcomes. Finally, the authors suggest that the Canadian Society of Hospital Pharmacists work with practitioners and faculties of pharmacy to develop best practices for student training.

All of these are important considerations, which, if in place, could make graduates practice-ready to provide direct patient care. They also ask that students become important and contributing members of the health care team and take responsibility for their own patients.

Overall, the profession and educational institutions will likely support the principles outlined by Hall and others. However, there will be challenges. The training of students in pharmacy is not financially supported by the government to the same extent as is the case for other health care disciplines. We also need to change the organizational culture of faculties of pharmacy and the mindset of preceptors, to ensure that they are ready and willing to take on this significant change in how training is administered. Such culture change may mean allowing students to learn from their errors early on, while ensuring that patient care is not compromised. We will need to expand the preceptor pool and provide support for preceptor development. Finally, hospital pharmacists will need to work closely with the educational institutions that are ultimately responsible for program delivery, to ensure that graduates develop the required competencies.

Discussions facilitated through the Blueprint for Pharmacy project have already started addressing some of these issues at a national level. In addition, some faculties of pharmacy have started to bring local hospital partners together to address issues related to training students. Faculties and the profession need to evaluate the services that students provide, to demonstrate their impact both on patient outcomes and on the ability of sites to increase provision of pharmacist services. Mechanisms should also be put into place to partner with other health systems and community sites and engage them in the new training models for pharmacy students. Developing "communities of practice" to provide a variety of training to students may enable better sharing of resources. Finally, we need to work with government to advocate for financial support in training pharmacist students.

Educational institutions, hospital and community pharmacies, and preceptors should consider how best to adapt the guiding principles outlined by Hall and others. ${ }^{9}$ Just as the need for more pharmacists to take on direct patient care responsibilities is urgent, so too is the need for institutions, community partners, and academia to work together on this joint vision to help future graduates become effective direct patient care providers.

\section{References}

1. Blueprint for Pharmacy: vision for pharmacy. Ottawa (ON): Canadian Pharmacists Association; 2012 [cited 2012 May 27]. Available from: www.pharmacists.ca/index.cfm/pharmacy-in-canada/blueprint-for-pharmacy/

2. Cipolle RJ, Strand LM, Morley PC. Pharmaceutical care practice: the clinician's guide. 2nd ed. New York (NY): McGraw-Hill Companies, Inc; 2004.

3. Baker GR, Norton PG, Flintoft V, Blais R, Brown A, Cox J, et al. 2004. The Canadian Adverse Events Study: the incidence of adverse events among hospital patients in Canada. CMAJ 2004;170(11):1678-1686.

4. Nigam R, Mackinnon NJ, U D, Hartnell NR, Levy AR, Gurnham ME, et al. Development of Canadian safety indicators for medication use. Healthc Q 2008;11(3 Spec No.):47-53.

5. Population projections for Canada, provinces and territories 2009 to 2036. Ottawa (ON): Statistics Canada; 2010 [cited 2012 May 27]. Cat. No. 91-520-X. Available from: www.statcan.gc.ca/pub/91-520-x/91-520x2010001-eng.pdf

6. Position statement and joint resolution on the Doctor of Pharmacy (PharmD) for the first professional degree at universities in Canada. Association of Faculties of Pharmacy of Canada and Association of Deans of Pharmacy of Canada; 2010 Feb [cited 2012 May 17]. Available from: www.afpc.info/downloads/1/AFPC_ADPC_PharmD_Position_ Statement_Resolution_Sept_2010.pdf

7. Educational outcomes for first professional degree programs in pharmacy (entryto-practice pharmacy programs) in Canada. Vancouver (BC): Association of Faculties of Pharmacy of Canada; 2010 Jun 3 [cited 2012 May 26]. Available from: http://afpc.info/downloads/1/AFPC_Education Outcomes_AGM_June_2010.pdf

8. Ashby DM. Permission granted. Am J Health Syst Pharm 2011;68(16): 1497-1504.

9. Hall K, Musing E, Miller DA, Tisdale JE. Experiential training for pharmacy students: time for a new approach. Can J Hosp Pharm 2012;65(4):285-293.

Lalitha Raman-Wilms, BSc(Phm), PharmD, FCSHP, is an Associate Professor and Associate Dean, Professional Programs, Leslie Dan Faculty of Pharmacy, University of Toronto, Toronto, Ontario. She is also an Associate Editor for the CJHP and a member of the Board of the Canadian Council for Accreditation of Pharmacy Programs.

\section{Address correspondence to:}

Dr Lalitha Raman-Wilms

Leslie Dan Faculty of Pharmacy

University of Toronto

144 College Street

Toronto ON M5S 3M2

e-mail: I.raman.wilms.a@utoronto.ca 